\title{
Is remnant liver volume ratio less than $30 \%$ still contraindication for living donor right hepatectomy?
}

\author{
Joo Dong Kim, Dong Lak Choi
}

Department of Surgery, Catholic University of Daegu School of Medicine, Daegu, Korea

Background: The lowest limits for a safe remnant/total volume ratio (RTVR) for living donor right hepatectomy (LDRH) is still not clear. Most centers have followed at least $30 \%$ for RTVR based on their experiences to keep donor safety. Recently, some centers reported that extended resection with RTVR less than $30 \%$ for LDRH and therefore, we describe our center's experience for LDRH with RTVR $<30 \%$ and evaluate the outcomes of living liver donors under these extended criteria.

Methods: We retrospectively reviewed the outcomes of 473 LDRHs which performed at our institution from January 2010 to December 2020. We performed right hepatectomy for 41 living donors with RTVR $<30 \%$ under the following criteria: age $\leq 40$, preservation of middle hepatic vein, no or minimal fatty changes ( $<15 \%)$, flat fish shaped left hemiliver, and RTVR $>25 \%$ and future remnant liver volume/body weight $\geq 0.45$. The outcomes in these extended living donors were compared with those in living donors under conventional criteria.

Results: The mean RTVR is $27.6 \% \pm 1.2 \%(25.5 \%-28.9 \%)$ in extended donor group and posthepatectomy liver failure (PHLF) occurred in 50 donors (10.6\%) and most cases were grade A except one case and no clinically significant PHLF was not evident for these extended criteria group. PHLF and major complications were not more frequent in living donors with RTVR $<30 \%$. In multivariate analysis, the only event for major complications was associated with PHLF but RTVR less than $30 \%$ was not related to PHLF. Moreover, in subgroup analysis with conventional donor group with same age and steatosis criteria, the evidence for PHLF and major complication rate were not different between the two groups.

Conclusions: LDRH under our extended criteria could be performed safely in donors with RTVR $<30 \%$ under our strict criteria when no other donors are available.

Corresponding author: Joo Dong Kim

E-mail: milledr1127@gmail.com

(c) The Korean Society for Transplantation

This is an Open Access article distributed under the terms of the Creative Commons Attribution Non-Commercial License (http://creativecommons.org/licenses/by-nc/4.0/) which permits unrestricted non-commercial use, distribution, and reproduction in any medium, provided the original work is properly cited. 